\title{
A Miniaturized Dual-Mode Bandpass Filter Using Slot Spurline Technique
}

\author{
Haiwen Liu, ${ }^{1,2}$ Jiuhuai Lei, ${ }^{2}$ Jing Wan, ${ }^{2}$ Yan Wang, ${ }^{2}$ Feng Yang, ${ }^{1}$ and Suping Peng ${ }^{1}$ \\ ${ }^{1}$ State Key Laboratory of Coal Resources and Safe Mining, China University of Mining \& Technology, Beijing 100083, China \\ ${ }^{2}$ School of Information Engineering, East China Jiaotong University, No. 808, East Shuanggang Road, Nanchang 330013, China \\ Correspondence should be addressed to Haiwen Liu; liuhaiwen@gmail.com
}

Received 19 February 2013; Revised 21 March 2013; Accepted 16 April 2013

Academic Editor: Amin Abbosh

Copyright (c) 2013 Haiwen Liu et al. This is an open access article distributed under the Creative Commons Attribution License, which permits unrestricted use, distribution, and reproduction in any medium, provided the original work is properly cited.

\begin{abstract}
A miniaturized dual-mode bandpass filter (BPF) with elliptic function response using slot spurline is designed in this paper. The slot spurline can not only splits the degenerate modes but also determine the type of filter characteristic (Chebyshev or elliptic). To miniaturize the resonator, four sagittate stubs are proposed. For demonstration purpose, a BPF operating at $5.75 \mathrm{GHz}$ for WLAN application was designed, fabricated, and measured. The measured results are in good agreement with the full-wave simulation results.
\end{abstract}

\section{Introduction}

Dual-mode microstrip filters have been widely used in wireless communications systems because of their advantages in microwave applications requiring high quality narrowband BPFs with features such as compact size, low cost, and low loss [1-3]. The concept of the dual-mode is based on coupling of the two degenerate modes in a geometrically symmetrical resonator, which is achieved by adding a perturbation element to split the two orthogonal modes. Many research works have been done on microstrip circular ring, square loop, circular disk, and square patch. For example, miniature meander loop resonator structures with microstrip cut perturbation for a dual-mode filter is reported in $[4,5]$. In [6], a novel dual-mode resonator with four arrow-shaped patches was proposed. Some modified waveguide structures based on defect ground structure (DGS) are reported to realize bandpass filter (BPF), such as slotline with DGS stubs, defected hairpin resonator, open-loop DGS, and defected SIR $[7,8]$. These BPFs with low cost and easy fabrication exhibit excellent bandpass performances. However, dualmode characteristics of these waveguide structures are not mentioned for circuit synthesis. In our previous work [9], a pentagonal slotline resonator was proposed. The strength and nature of the coupling between the degenerate modes can be adjusted by the defected corner cut perturbation. The drawback of this resonator is that it has a large circuit size. In [10], a dual-mode bandpass filter using slotline resonator and stubs was designed. The resonant frequency of this resonator was reduced about $7 \%$ by loading slotline stubs.

Based on our previous work in dual-mode slotline resonator $[9,10]$, a miniaturized dual-mode BPF using slotline resonator, slot spurlines, and sagittate stubs is proposed in this paper. The type of filter characteristic (Chebyshev or elliptic) can be controlled by the location of the spurlines. However, in references $[9,10]$, the type of filter characteristic is adjusted by changing the perturbation's size. In this paper, the filter can be miniaturized to a great extent by using the sagittate stubs. The proposed resonator in this work has a larger size reduction compared to the resonators in $[9,10]$. The miniaturization technique is discussed in detail. Finally, this filter with elliptic characteristics is designed and fabricated. Measurements and simulations are given.

\section{Miniaturization Technique}

A conventional square resonator consists of four $\lambda / 4$ transmission lines with the same characteristic impedances, and its size is determined by the guided wavelength of the transmission line. It is possible to reduce the physical length of a transmission line by using a $\pi$ equivalent topology. 


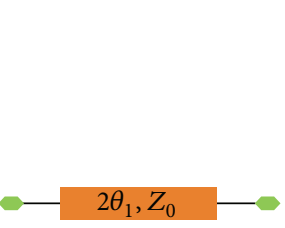

(a)

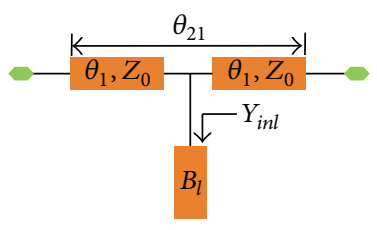

(b)
FIgURE 1: (a) Conventional transmission line. (b) The lengthreduced transmission line with a reactance part.

Figure 1(a) shows a transmission line with characteristic impedance $Z_{0}$ and electrical length $2 \theta_{1}$, whereas Figure 1(b) shows a transmission line $\left(2 \theta_{1}, Z_{0}\right)$ loaded by a stub with reactance $B_{l}$. The admittance of the reactance is marked with $Y_{i n l}$. To demonstrate size reduction of the transmission line, $\mathrm{ABCD}$ matrix is used to analyze equivalent circuit model. The transmission characteristics of the equivalent circuit can be presented by ABCD matrix that normalized to characteristic admittance $Y_{0}$. Let characteristic impedance $Z_{0}=1 / Y_{0}$ and the normalized admittance $\bar{Y}_{\text {inl }}=Y_{\text {inl }} / Y_{0}$; the normalized $A B C D$ matrix is expressed as follows:

$$
\begin{aligned}
& {\left[\begin{array}{ll}
A & B \\
C & D
\end{array}\right]} \\
& =\left[\begin{array}{cc}
\cos \theta_{1} & j \sin \theta_{1} \\
j \sin \theta_{1} & \cos \theta_{1}
\end{array}\right] \cdot\left[\begin{array}{cc}
1 & 0 \\
\overline{Y_{i n l}} & 1
\end{array}\right] \cdot\left[\begin{array}{cc}
\cos \theta_{1} & j \sin \theta_{1} \\
j \sin \theta_{1} & \cos \theta_{1}
\end{array}\right] \\
& =\left[\begin{array}{cc}
\cos 2 \theta_{1}+\left(\frac{j}{2}\right) \cdot \overline{Y_{i n l}} \cdot \sin 2 \theta_{1} & j \sin 2 \theta_{1}-\overline{Y_{i n l}} \cdot \sin ^{2} \theta_{1} \\
j \sin 2 \theta_{1}+\overline{Y_{i n l}} \cdot \cos ^{2} \theta_{1} & \cos 2 \theta_{1}+\left(\frac{j}{2}\right) \cdot \overline{Y_{i n l}} \cdot \sin 2 \theta_{1}
\end{array}\right] .
\end{aligned}
$$

Then, the $S$-parameter of the length-reduced transmission line can be obtained by matrix conversion as follows:

$$
S_{21}=\frac{2}{A+B+C+D}
$$

By substituting (1) into (2), we can get

$$
\left|S_{21}\right|=\left|\frac{2}{2+\overline{Y_{\text {inl }}}}\right| \text {. }
$$

The total electrical length of the length-reduced transmission line can be obtained by matrix conversion as follows:

$$
\theta_{21}=\arctan \left(\frac{\tan 2 \theta_{1}-(j / 2) \cdot \overline{Y_{i n l}}}{1+(j / 2) \cdot \overline{Y_{i n l}} \cdot \tan 2 \theta_{1}}\right)
$$

For getting a real $\theta_{21}, Y_{i n l}$ must be a pure imaginary in (4). So, let $Y_{\text {inl }}=j B Y_{0}$ and formula (4) can be simplified as

$$
\theta_{21}=2 \theta_{1}+\arctan \left(\frac{B}{2}\right)
$$

It can be found from (5) that when $B>0\left(Y_{\text {inl }}>0\right)$, the absolute value of the $\theta_{21}$ is larger than that of $2 \theta_{1}$. The

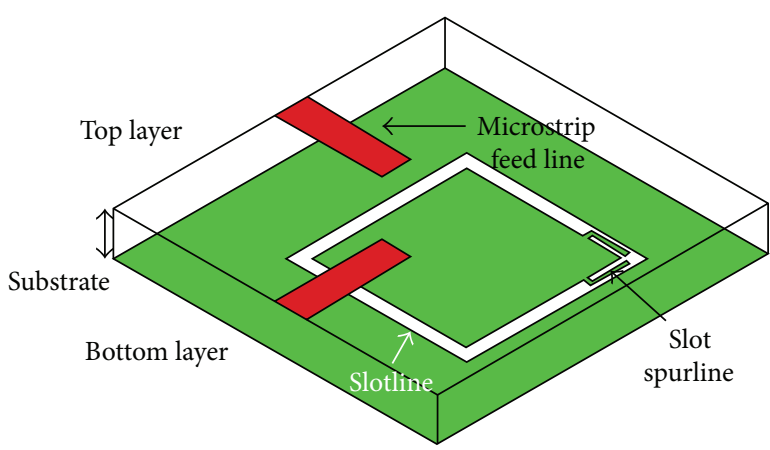

FIgURE 2: Three-dimensional view of a slotline square resonator with a pair of slot spurlines.

fact implies that the physical length of a transmission line can be reduced by a reactance center loaded effectively. Thus, compactness of the structure is achieved.

\section{Dual-Mode Slot Filter Design}

Figure 2 shows the three-dimensional view of a slotline structure. It consists of two layers. On the bottom layer, a fullwavelength $(\lambda)$ slot square resonator with a pair slot spurlines is etched on the backside metallic ground plane. On the top layer, there is a pair of microstrip $50 \Omega$ feed lines.

Using spurlines perturbation to excite the degenerate modes of microstrip square loop resonator is discussed in this paper. A pair of spurlines are located in different corners, the nature of the coupling between the degenerate modes will present inductive (magnetic) or capacitive (electric), and the frequency response characteristic will exhibit a Chebyshev characteristic or an elliptic characteristic, respectively. Just like microstrip square loop resonator, two different dualmode slotline resonators with slot spurlines are shown in Figures 3(a) and 3(b). The two dual-mode slotline resonators were simulated by Ansoft HFSS 10 and the results are given in Figure 3(c). When the spurlines are embedded on the corners A and C, seen in Figure 3(b), it exhibits a Chebyshev characteristic. However, when the spurlines are embedded on the corner B, seen in Figure 3(a), it exhibits an elliptic characteristic, as confirmed by our simulations shown in Figure 3(c). The reason is that the slot spurlines perturbation's location can change the nature of the coupling.

Current distributions of the dual-mode resonator are depicted in Figures 4 and 5. Results show that the locations of the higher and the lower density $\mathrm{H}$-field distribution regions of Mode-II $\left(f_{2}\right)$ are rotated by 90 degrees from those of ModeI $\left(f_{1}\right)$.

Based on the afore-mentioned analysis, a miniaturized dual-mode slot filter is proposed by using a loaded stub. The three-dimensional view of the miniaturized square resonator is shown in Figure 6. The top view is shown in Figure 7. There is a sagittate stub center loaded on each side of the square slot resonator. The sagittate stub has the advantage of making maximum use of the inner space of the square resonator. Therefore, the sagittate stub can be used to reduce the size of the square resonator to a great extent. 


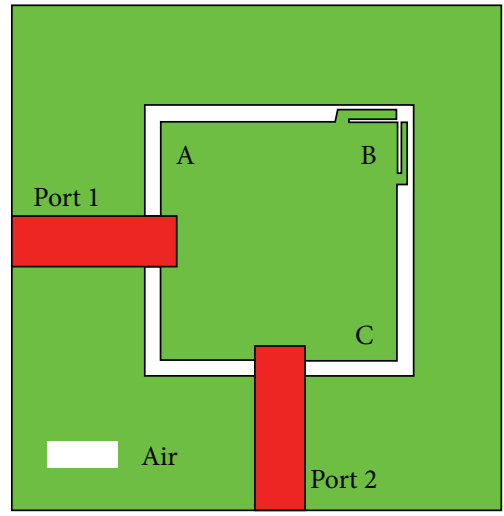

(a)

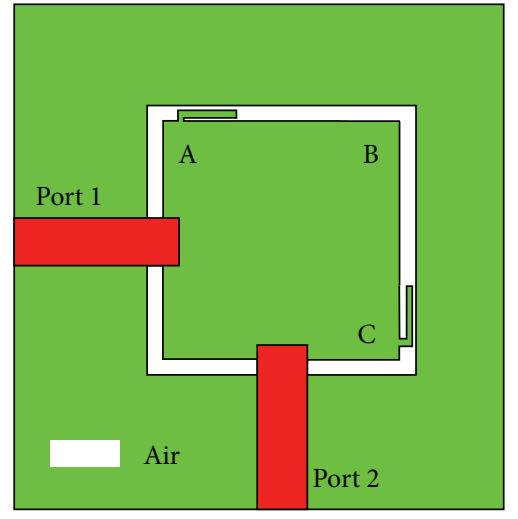

(b)

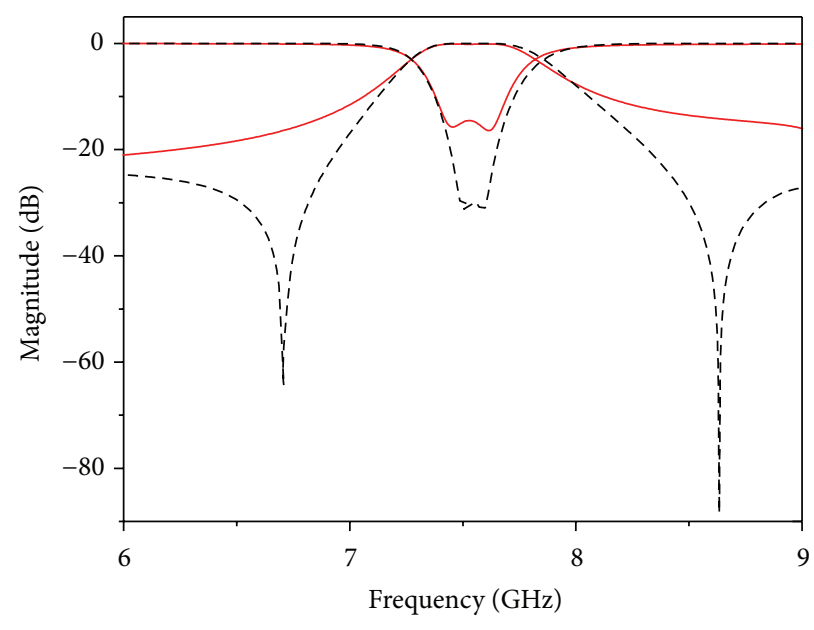

On corner A and C

--- - On corner B

(c)

FIGURE 3: (a) Dual-mode square slotline resonator with the slot spurlines on corner B. (b) Dual-mode square slotline resonator with the slot spurlines on corners A and C. (c) Their simulated frequency response, where the black dash line is for (a) and the red line is for (b).

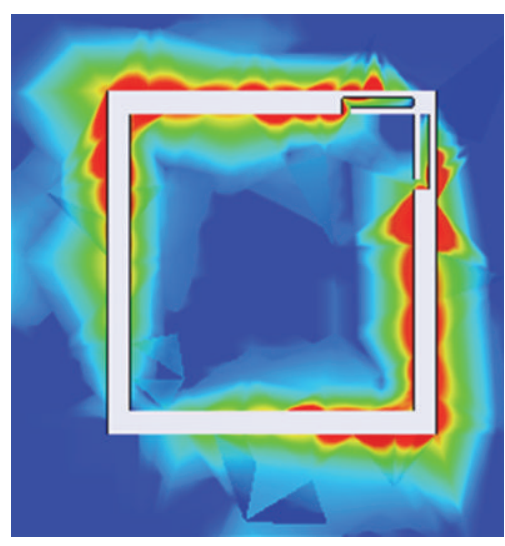

(a)

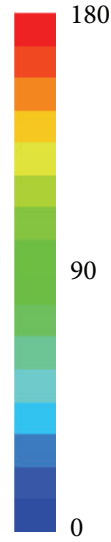

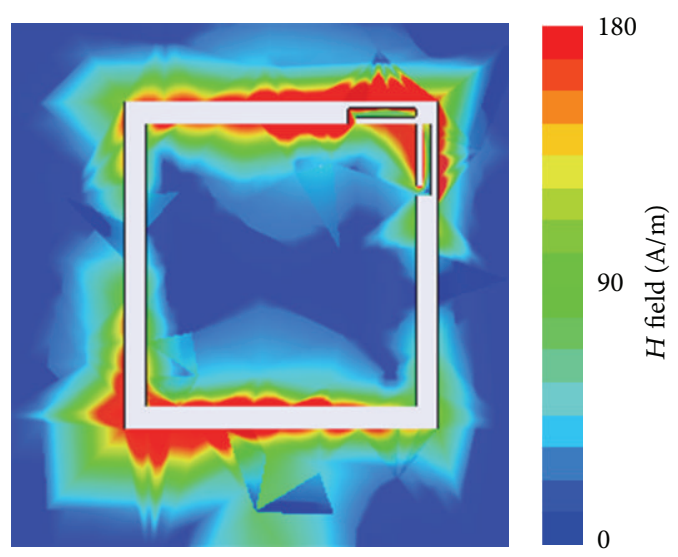

(b)

FIGURE 4: Simulated current distributions for two degenerate modes with an elliptic characteristic (spurlines on corner B): (a) $f_{1}=7.5 \mathrm{GHz}$; (b) $f_{2}=7.59 \mathrm{GHz}$. 


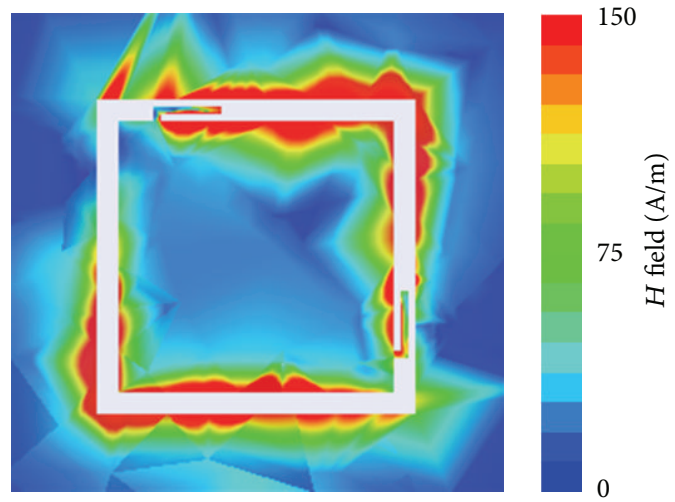

(a)

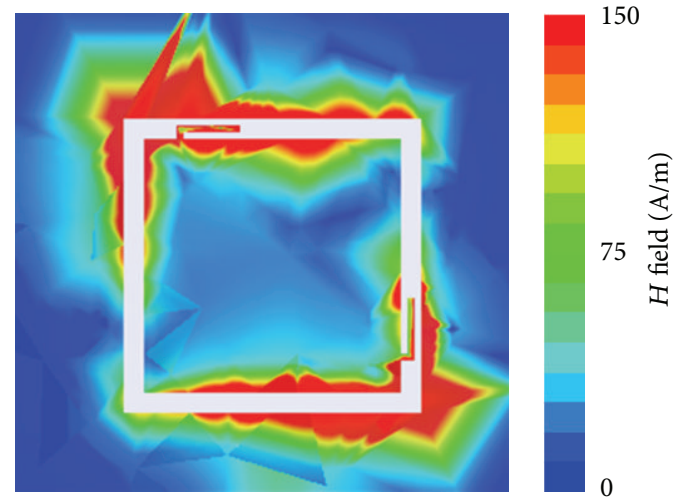

(b)

FIgURE 5: Simulated current distributions for two degenerate modes with a Chebyshev characteristic (spurline on corners A and C): (a) $f_{1}=7.45 \mathrm{GHz}$; (b) $f_{2}=7.63 \mathrm{GHz}$.

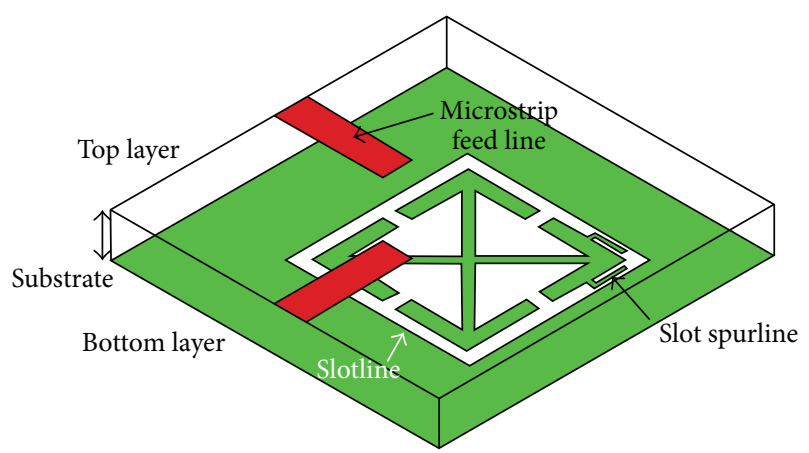

FIGURE 6: The three-dimensional view of the miniaturized square resonator.

\section{Measurements}

For demonstration purpose, a miniaturized dual-mode BPF operating at $5.75 \mathrm{GHz}$ for WLAN application was designed. The designed filter is designed on a Taconic RF-35A2 substrate with a relative dielectric constant of 3.5 , a thickness of $0.76 \mathrm{~mm}$, and a loss tangent of 0.0011 . Following the afore-mentioned design method, the dimensions are given as follows: $w_{1}=0.6 \mathrm{~mm}, w_{2}=0.2 \mathrm{~mm}, L_{1}=8.4 \mathrm{~mm}, L_{2}=$ $0.8 \mathrm{~mm}, L_{3}=0.3 \mathrm{~mm}$, and $L_{4}=2.3 \mathrm{~mm}$. The overall size of this filter is $8.4 \times 8.4 \mathrm{~mm}^{2}$ (about $0.175 \lambda_{g}$ by $0.175 \lambda_{g}$, where $\lambda_{g}$ is the guided wavelength at the center frequency of the passband). The size of the conventional resonator at $5.75 \mathrm{GHz}$ is $12 \times 12 \mathrm{~mm}^{2}$. It means that the proposed resonator has a size reduction of $30 \%$ compared to the conventional resonator. The simulated results are illustrated in Figure 10. Current distributions of the miniaturized dual-mode resonator are depicted in Figure 8 . The phase difference of the high-density distributions between two degenerate modes is about $90^{\circ}$. This is consistent with the previous analysis.

Figure 9 shows the photographs of the proposed miniaturized dual-mode slot bandpass filter. The measured results are illustrated in Figure 10. Results show that the passband of

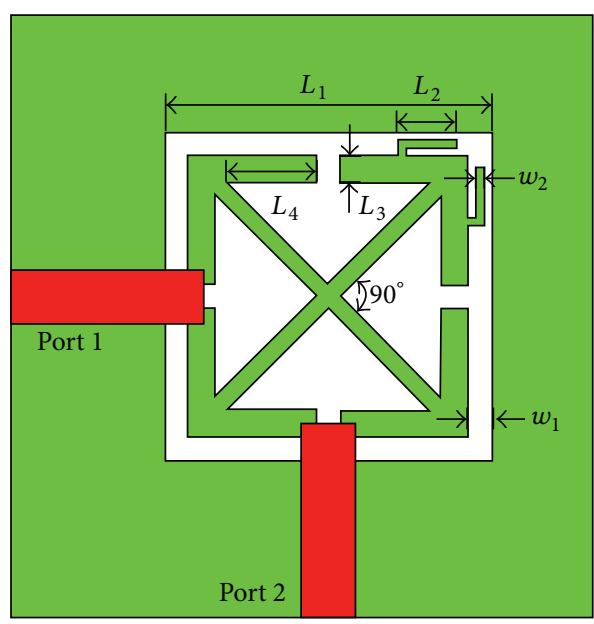

FIGURE 7: Top view of the proposed $t$ miniaturized dual-mode square filter.

the filter is centered at $5.785 \mathrm{GHz}$ with the fractional bandwidth of $4 \%$. The minimum insertion loss of the measured result is $1.93 \mathrm{~dB}$ at the passband. The insertion loss is mainly due to radiation loss of the slotline. In addition, there are two transmission zeros on both sides of the passbands. They are $-61.2 \mathrm{~dB} /-78.7 \mathrm{~dB}$ at frequencies of $5.44 \mathrm{GHz} / 6.56 \mathrm{GHz}$ respectively. They are close to the passband edges and can greatly improve the selectivity and stopband suppression. The transition is usually required to have a flat group delay [11]. Figure 11 illustrates the group delays of the dual-mode filter. The measured result shows that the filter has about 0.45 ns peak-to-peak variation in the group delay. This raised variation is caused by a tradeoff between rejection skirt in $\mathrm{S}_{21}$-magnitude and linearity in phase [12]. Measured result agrees well with the simulated one and proves the validity of the introduced design principles to produce the dual-mode characteristics. 


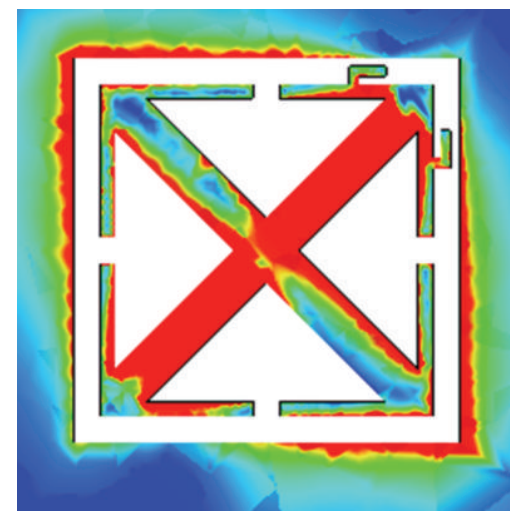

(a)

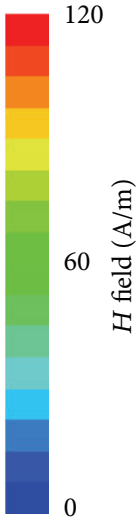

0

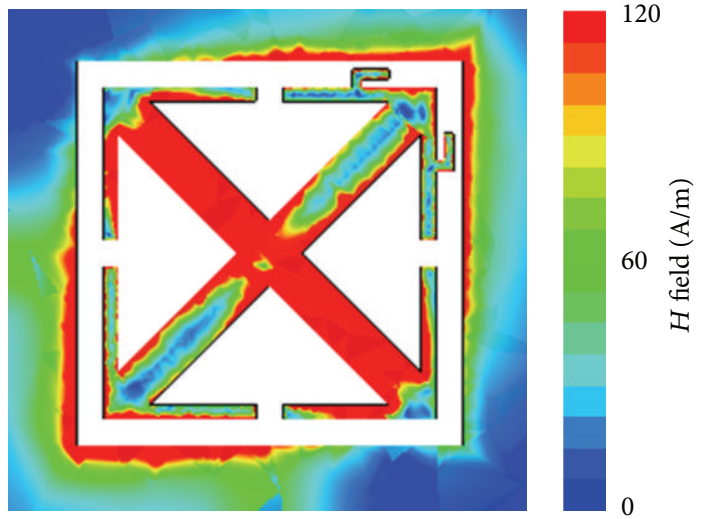

(b)

FigURE 8: Simulated current distributions of the final filter: (a) $f_{1}=5.77 \mathrm{GHz}$; (b) $f_{2}=5.8 \mathrm{GHz}$.

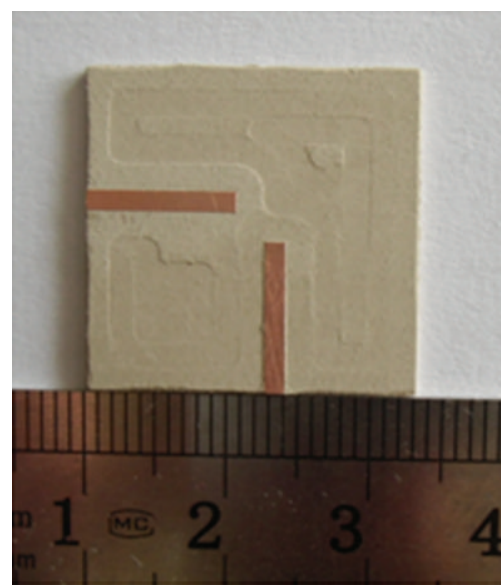

(a)

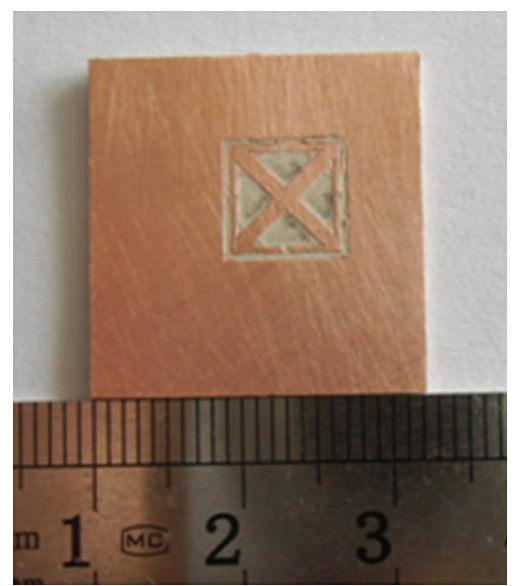

(b)

Figure 9: Photographs of proposed filter: (a) top view and (b) bottom view.

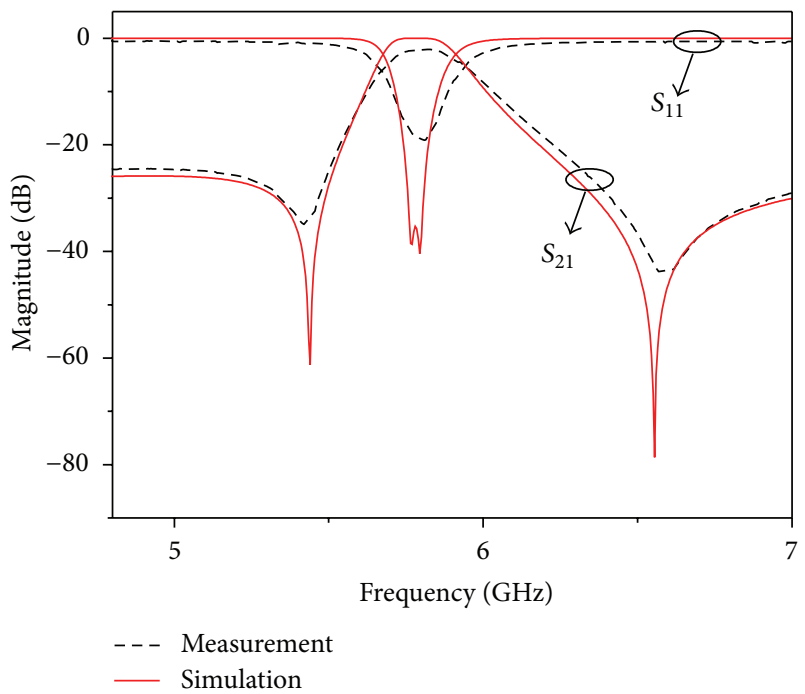

FIGURE 10: Measured and simulated frequency responses of the proposed dual-mode bandpass filter.

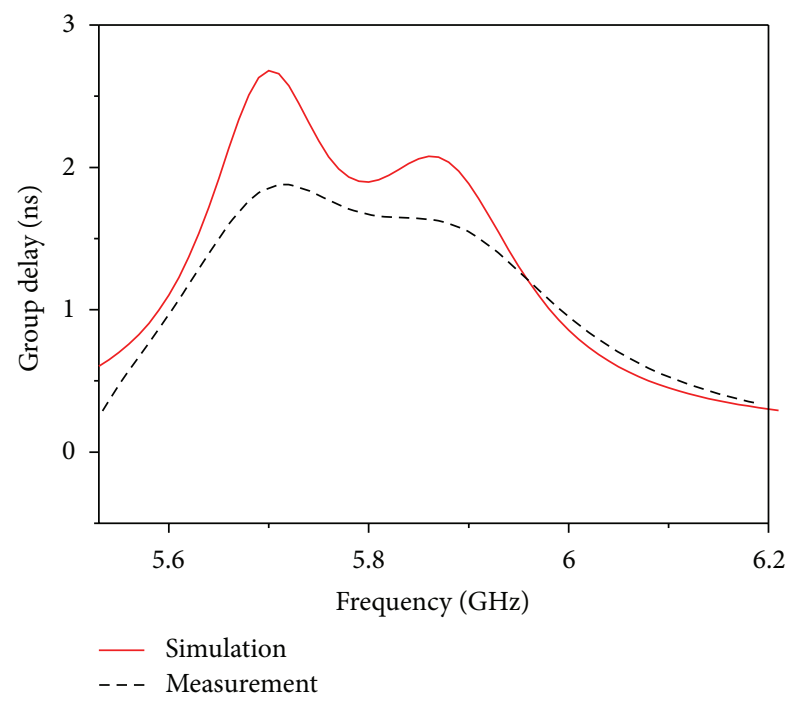

FIGURE 11: Group delay of the proposed miniaturized filter. 


\section{Conclusion}

A miniaturized dual-mode slot bandpass filter using slot spurline technique is presented in this paper. The type of filter characteristic (Chebyshev or elliptic) can be controlled by the location of the spurlines. Also, the miniaturization technique is studied. The sagittate stub can make full use of the inner space of the square resonator. Therefore, the filter can be miniaturized to a great extent. A good agreement between the simulated and experimental responses is obtained and demonstrated. The presented compact dual-mode filter is particularly suitable for communication systems.

\section{Acknowledgments}

This work was supported by the National Science Foundation of China (no. 61061001) and International Cooperation Funds and Science and Technology Innovation Team of Jiangxi Province of China (nos. 20121BDH80015 and 20122BCB24025).

\section{References}

[1] S. Amari, "Application of representation theory to dual-mode microwave bandpass filters," IEEE Transactions on Microwave Theory and Techniques, vol. 57, no. 2, pp. 430-441, 2009.

[2] S. W. Ren, H. L. Peng, J. F. Mao, and A. M. Gao, "Compact quasielliptic wideband bandpass filter using cross-coupled multiplemode resonator," IEEE Microwave and Wireless Components Letters, vol. 22, pp. 397-399, 2012.

[3] A. Görür, "Description of coupling between degenerate modes of a dual-mode microstrip loop resonator using a novel perturbation arrangement and its dual-mode bandpass filter applications," IEEE Transactions on Microwave Theory and Techniques, vol. 52, no. 2, pp. 671-677, 2004.

[4] A. Görür and C. Karpuz, "Miniature dual-mode microstrip filters," IEEE Microwave and Wireless Components Letters, vol. 17, no. 1, pp. 37-39, 2007.

[5] R. Chu, J. F. Mao, and L. Zhou, "A meandering dual-mode bandpass filter with rectangular-loop perturbation," Microwave and Optical Technology Letters, vol. 52, no. 10, pp. 2356-2359, 2010.

[6] S. Fu, B. Wu, J. Chen, S. J. Sun, and C. H. Liang, "Novel secondorder dual-mode dual-band filters using capacitance loaded square loop resonator," IEEE Transactions on Microwave Theory and Techniques, vol. 60, no. 3, pp. 477-483, 2012.

[7] W. Feng, K. Deng, W. Che, and S. Dong, "Novel microstrip bandpass filter based on defected ground structure and slotline coupling techniques," Microwave and Optical Technology Letters, vol. 52, no. 1, pp. 4-6, 2010.

[8] Z. Q. Cheng, J. Li, X. G. Mao, S. Tan, and J. K. Chen, "Plip-chip intergrated Oscillator with reduced phase noise and enhanced output power by using DGS," Journal of Infrared and Millimeter Waves, vol. 27, no. 6, pp. 401-404, 2008.

[9] H. W. Liu, L. Shen, Z. C. Zhang, J. S. Lim, and D. Ahn, "Dual-mode dual-band bandpass filter using defected ground waveguide," Electronics Letters, vol. 46, no. 13, pp. 895-897, 2010.

[10] H. W. Liu, Y. C. Fan, G. W. Wei et al., "Compact dual- mode bandpass filter using slotline resonator and stubs," Microwave and Optical Technology Letters, vol. 54, no. 3, pp. 641-644, 2012.
[11] A. M. Abbosh, "Multioctave microstrip-to-coplanar waveguide vertical transition," Microwave and Optical Technology Letters, vol. 53, no. 1, pp. 187-189, 2011.

[12] R. Li and L. Zhu, "Compact UWB bandpass filter using stubloaded multiple-mode resonator," IEEE Microwave and Wireless Components Letters, vol. 17, no. 1, pp. 40-42, 2007. 

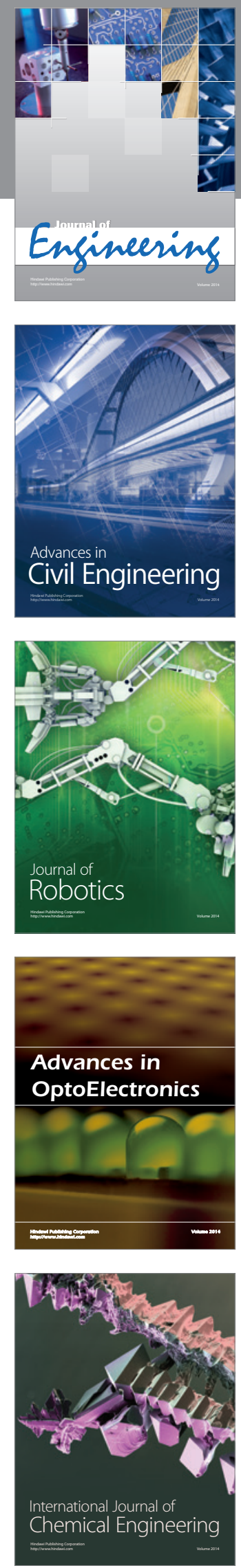

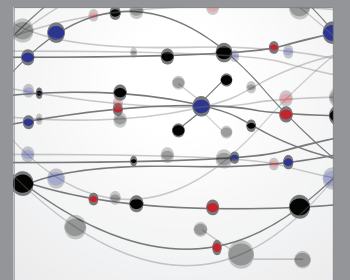

The Scientific World Journal
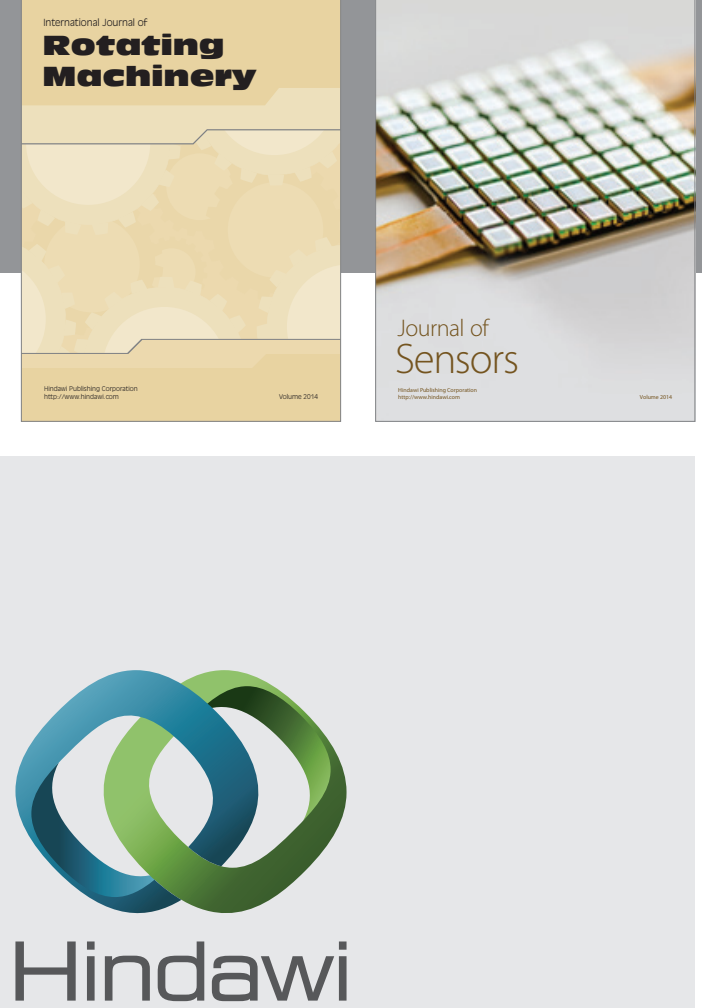

Submit your manuscripts at http://www.hindawi.com
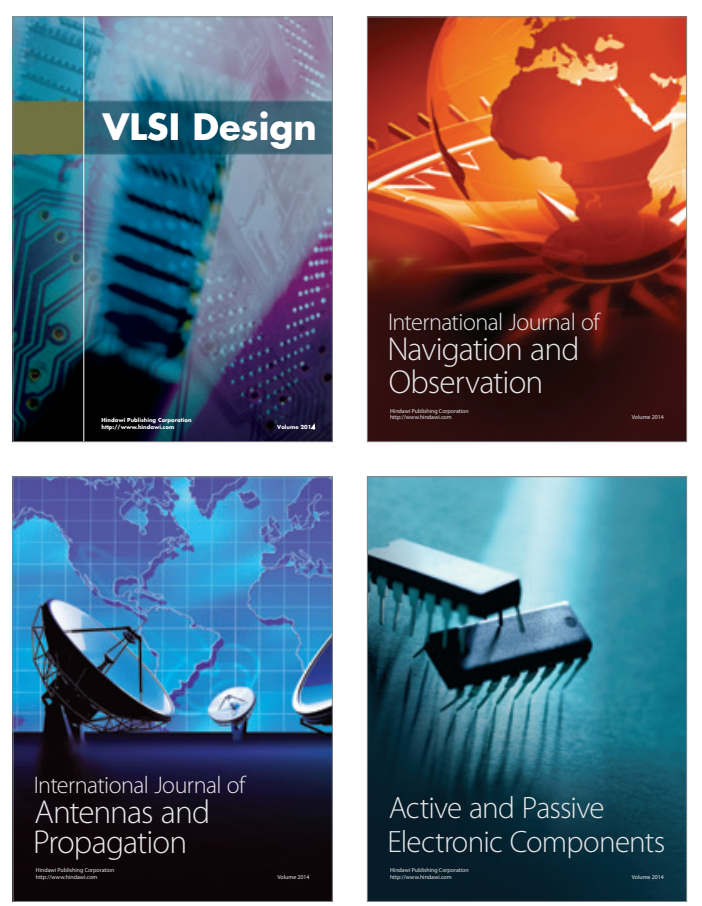
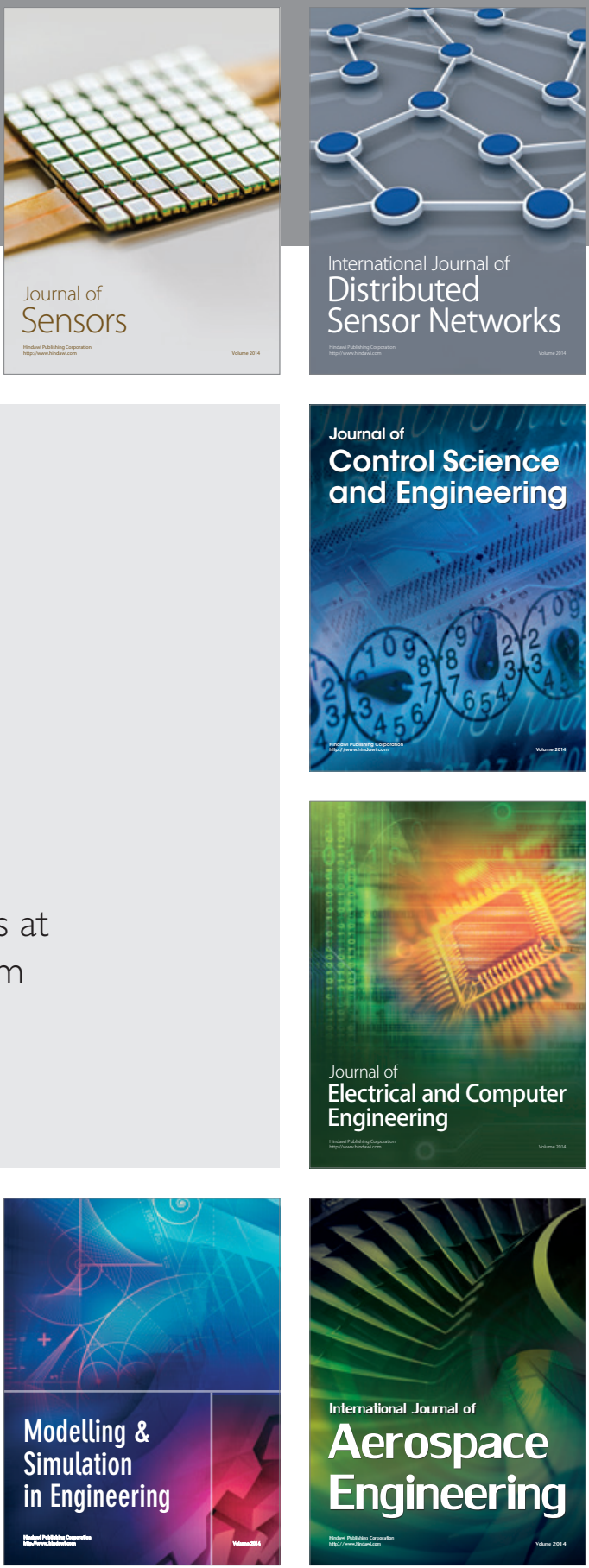

Journal of

Control Science

and Engineering
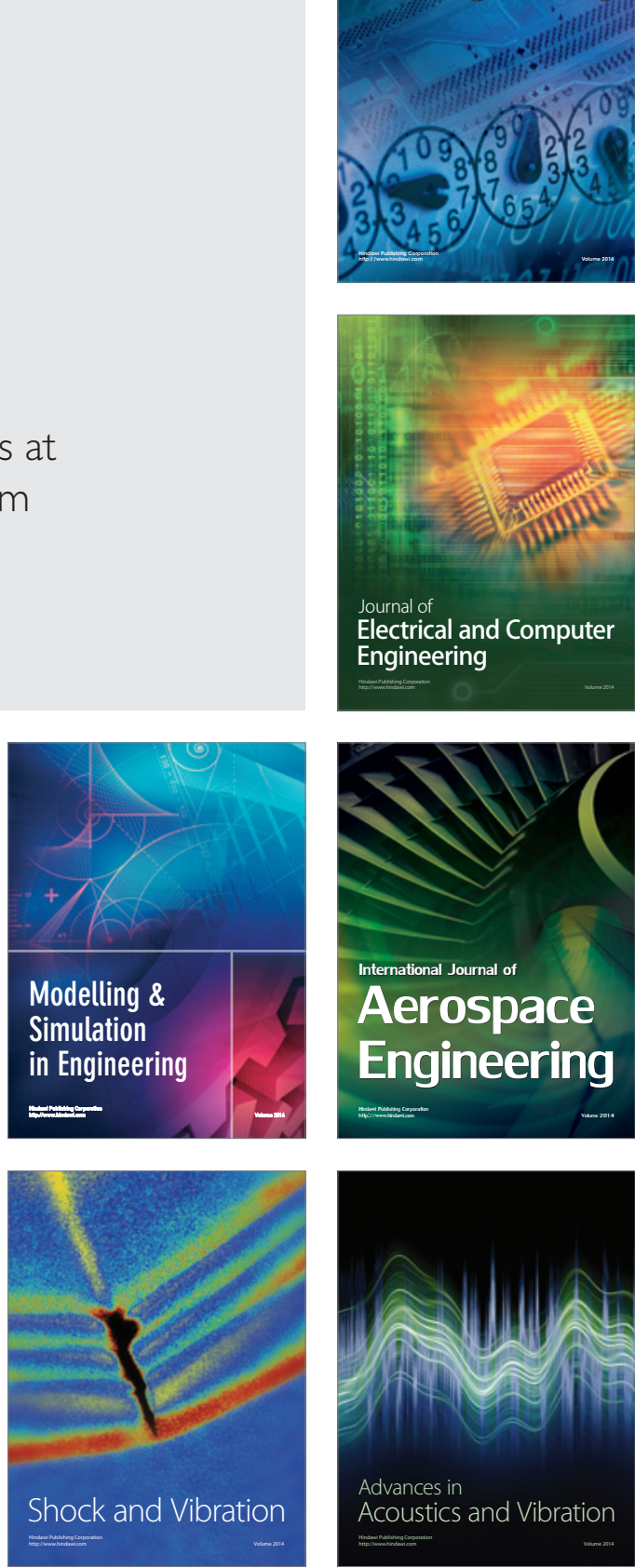